\title{
RELAXATION OF THE CYCLOTRON SPIN-FLIP EXCITATION IN A SPIN-UNPOLARIZED QUANTUM HALL SYSTEM
}

\author{
S. Dickmann \\ Institute for Solid State Physics of RAS, 142432 Chernogolovka, Moscow District, Russia \\ E-mail: dickmann@mail.ru
}

Received 20 April 2012; accepted 7 June 2012

\begin{abstract}
Cyclotron spin-flip excitation in an even-integer quantum Hall system is the lowest-energy excitation separated from the ground state by the gap slightly smaller than the cyclotron energy, and from the upper magnetoplasma excitation by the Coulomb gap [1. 2]. At the filling factor $\nu=2$ in a real system these gaps are in the vicinity of $10 \mathrm{meV}$ and $0.5 \mathrm{meV}$ and thus are much larger than the Zeeman gap $(\sim 0.1 \mathrm{meV})$ and temperature $(<0.01 \mathrm{meV})$ respectively. Under these conditions the cyclotron spin flip excitation can not relax in a purely electronic way but only with emission of a short-wave phonon $\left(k \sim 310^{7} / \mathrm{cm}\right)$. As a result the relaxation in a modern wide-thickness quantum well occurs very slowly, the characteristic relaxation time being $\sim 1 \mathrm{~s}$. The cyclotron spin-flip excitation in the narrow vicinity of filling factors $\nu=2,4, \ldots$ should therefore be considered as a collective metastable excitation in the quantum Hall system.
\end{abstract}

Keywords: quantum Hall system, spin flip, cyclotron mode, spin relaxation, metastable quantum state, exciton condensate, qubit realization

PACS: 73.21.Fg, 73.43.Lp, 78.67.De

Growing interest in the problem of excitation lifetimes in low-dimensional electron systems has been observed for at least two last decades. Particularly a two-dimensional (2D) electron gas under quantum Hall conditions represents an object of these investigations, but all recent studies in this specific field have been in fact devoted to spin relaxation in a spin-polarized system (in other words, in the quantum Hall ferromagnet, see Refs. [3-7] and references therein). These researches are basically related to relaxation of lowest energy excitations - spin waves separated from the ground state only by a narrow Zeeman gap, $\epsilon_{\mathrm{Z}}=$ $\left|g \mu_{B} B\right| \sim 1 \mathrm{~K}$, much smaller than the cyclotron energy, $\epsilon_{\mathrm{Z}} \ll \hbar \omega_{c}$. Due to this circumstance, elementary processes actually determining spin-wave annihilation/generaton can be purely electronic: loss of the Zeeman energy can be compensated by a change in the spin-wave "kinetic energy" originating from the $e-e$ correlation exchange [7,-9].

Now I report on a study of the lowest-excitation life-time in an unpolarized integer quantum Hall system. In this case the excitation represents also a spin flip but separated from the ground state by a wide gap which is only somewhat smaller than the cyclotron one. By contrast to the situation with quantum Hall ferromagnet, the cyclotron-energy loss is not compensated electronically. This could occur non-radiatively only due to the electron-phonon interaction leading to emission of acoustic phonons with "huge" energy $\approx \hbar \omega_{c}$. As a result, the relaxation turns out to be extremely slow - according to the presented calculation by 5-6 orders slower than the spin-wave relaxation in quantum Hall ferromagnet. The predicted relaxation time could compete or even be longer than the record spin relaxation time $T_{1} \sim 100 \mathrm{~ms}$, fixed experimentally at low magnetic fields in a single-electron quantum dot [10]. Meanwhile, as opposed to the spin relaxation in a quantum dot, relaxation of the $\nu=2$ lowest excitation in a quantum well should sharply decelerate with increasing magnetic field.

Spectrum of the lowest-energy excitation in an unpolarized integer quantum Hall system (specifically in the $\nu=2$ case) was studied earlier both theoretically [1, 11] and experimentally [2]. The obtained results allow to consider this state as a cyclotron spin-flip exciton (CSFE), i. e. a collective excitation where an electron is effectively promoted from a fully occupied Landau level to the next fully empty level with a spin flip (for reference, the spin wave in a quantum Hall ferromagnet is a spin exciton where an electron is promoted to the upper spin sublevel of the same Landau level). The CSFE represents a triplet with the $S=1$ and 
$S_{z}=0, \pm 1$ spin components separated by the Zeeman gap. Accordingly, the $S_{z}=1$ component is the lowestenergy excitation. By analogy with previous works devoted to exciton spectra calculations [1, 12, 13] as well as to the exciton relaxation [3, 7, 9, 14,-18], I employ the excitonic representation technique, where exciton states are used as a basis set instead of single electron Fermi states. The exciton states are generated by exciton creation operators, [19, 20]

$$
\mathcal{Q}_{a b \mathbf{q}}^{\dagger}=\frac{1}{\sqrt{\mathcal{N}_{\phi}}} \sum_{p} e^{-i q_{x} p} b_{p+\frac{q_{y}}{2}}^{\dagger} a_{p-\frac{q_{y}}{2}}
$$

acting on the ground state $|0\rangle$. In this definition $a_{p}$ and $b_{p}$ are electron Fermi annihilation operators corresponding to "initial" and "final" states of the promoted electron, $\mathbf{q}$ is the exciton dimensionless momentum. Index $p$ labels intrinsic Landau level states which have wave functions $\psi_{n p}(\mathbf{r})=\left(2 \pi \mathcal{N}_{\phi}\right)^{-1 / 4} e^{i p y} \varphi_{n}(p+x)$ in the Landau gauge. $\left[\varphi_{n}(x)\right.$ is the oscillator function, where $n$ is the Landau level number, $\mathcal{N}_{\phi}$ is the number of the magnetic flux quanta; in Eq. (1) and everywhere below we measure length in units of $l_{B}$, and wave vectors in units of $1 / l_{B}\left(l_{B}\right.$ being the magnetic length).] $a$ and $b$ are binary indexes indicating both the Landau level number and the spin state. For example, for the $S_{z}=1$ component of the CSFE at $\nu=2$, these are $a=(0, \downarrow) \equiv \overline{0}$ and $b=(1, \uparrow) \equiv 1$ ( $n$ and $\bar{n}$ are spin-up and spin-down sublevels of the $n$-th Landau level. The exciton operators on their own form a commutation Lie algebra, namely:

$$
\begin{aligned}
{\left[\hat{\mathcal{Q}}_{c d \mathbf{q}_{1}}^{\dagger}, \hat{\mathcal{Q}}_{a b \mathbf{q}_{2}}^{\dagger}\right] \equiv } & N_{\phi}^{-1 / 2}\left(e^{-i\left(\mathbf{q}_{1} \times \mathbf{q}_{2}\right)_{z} / 2} \delta_{b, c} \hat{\mathcal{Q}}_{a d \mathbf{q}_{1}+\mathbf{q}_{2}}^{\dagger}\right. \\
& \left.-e^{i\left(\mathbf{q}_{1} \times \mathbf{q}_{2}\right)_{z} / 2} \delta_{a, d} \hat{\mathcal{Q}}_{c b \mathbf{q}_{1}+\mathbf{q}_{2}}^{\dagger}\right),(2)
\end{aligned}
$$

where symbol $\delta$ is the Kronecker delta.

The basic property of the exciton states, $\mathcal{Q}_{a b \mathbf{q}}^{\dagger}|0\rangle$, consists in the fact that they diagonalize a considerable part in the exact many-electron Coulomb interaction Hamiltonian $H_{\text {int }}$. Sometimes these states even represent eigen states of the Hamiltonian, if the manyelectron Srödinger equation is solved perturbatively in terms of the small parameter $r_{\mathrm{s}}=\alpha\left(e^{2} / \kappa l_{B}\right) / \hbar \omega_{c}$. This situation, e.g., always takes place for lowest excitations at an integer filling - in particular, for the spin wave at $\nu=1$ and for the CSFE at $\nu=2 . r_{\mathrm{s}}$ is the ratio of the characteristic Coulomb interaction energy to the cyclotron gap ( $\kappa$ is the GaAs dielectric constant, and $\alpha<1$ is the averaged form-factor arising due to finiteness of the 2D electron-layer thickness). Besides, at $\nu=2$ the excitonic representation of the CSFE state,
$\mathcal{Q}_{\overline{0} 1, \mathbf{q}}^{\dagger}|0\rangle$, allows at zero $q$ to find first order corrections to the state and thus to calculate the CSFE energy up to second order in $r_{\mathrm{s}}$. Exactly this second-order result yields the leading contribution to the Coulomb energy in the case [1].

So, on the basis of the results [1, 2, 11] one can conclude that in the leading approximation in $q$ and $r_{\mathrm{s}}$ the CSFE spectrum is

$$
E\left(S_{z}, q\right)=\hbar \omega_{c}-\epsilon_{\mathrm{Z}} S_{z}-\Delta E_{\mathrm{C}}+q^{2} / 2 M_{\mathrm{x}},
$$

where the negative value $-\Delta E_{\mathrm{C}}$ is the Coulomb shift exactly calculated to second order in $r_{\mathrm{s}}$, namely in the $\nu=2$ case: [1]

$$
\Delta E_{\mathrm{C}}=\sum_{m=2}^{\infty} \frac{2-2^{2-m}}{m m !\left(m^{2}-1\right)} \int_{0}^{\infty} d p p^{2 m+1}[F(p)]^{2} e^{-p^{2}}
$$

in units of $2 \mathrm{Ry}=\left(e^{2} / \kappa l_{B}\right)^{2} / \hbar \omega_{c}=m_{e}^{*} e^{4} / \kappa^{2} \hbar^{2}$, and $1 / M_{\mathrm{x}}$ is the coefficient calculated to first order in $r_{\mathrm{s}}[11]$,

$$
1 / M_{\mathrm{x}}=\int_{0}^{\infty} d p F(p) e^{-p^{2} / 2}\left(p^{2} / 2-p^{4} / 4\right)
$$

in units of $e^{2} / \kappa l_{B} . \quad F(q)$ in Eqs. (4) and (5) is the finite thickness form-factor: $F(q)=\iint d z_{1} d z_{2}$ $\times e^{-q\left|z_{1}-z_{2}\right| / l_{B}}\left|\chi\left(z_{1}\right)\right|^{2}\left|\chi\left(z_{2}\right)\right|^{2}(\chi$ is the size-quantized wave-function of an electron confined in the $z$-direction). $\Delta E_{\mathrm{C}}$ is definitely positive value, whereas the sign of $1 / M_{\mathrm{x}}$ varies with $F(q)$. In the ideal 2D case $F(q)=1$, and $1 / M_{\mathrm{x}}$ is negative (though at $q \gtrsim 1$ $E\left(S_{z}, q\right)$ grows with $\left.q\right)$ [11]. In the case of modern wide quantum-well structures $F(q)$ is rapidly decreasing with $q$, and $1 / M_{\mathrm{x}}$ becomes positive. I will consider exactly this real situation, i. e. assume that $1 / M_{\mathrm{x}}>0$. In practice the $\Delta E_{\mathrm{C}}$ and $1 / M_{\mathrm{x}}$ values should be found from experimental data. For example, according to Ref. [2] one gets $\Delta E_{\mathrm{C}} \approx 0.35 \mathrm{meV}$. Yet, until now there are no direct measurements yielding $1 / M_{\mathrm{x}}$, and I am forced to estimate this with the help of Eq. (5) by using some semi-empiric function $F(p)$. Thus I find that $0.3 \mathrm{meV} \leq \Delta E_{\mathrm{C}}<1 / M_{\mathrm{x}} \leq 1 \mathrm{meV}$. Taking into account that $\epsilon_{\mathrm{Z}}=0.0255 B \mathrm{meV}$ and $\hbar \omega_{c}=1.73 B \mathrm{meV}$ ( $B$ is in Teslas), one can see that the first term in Eq. (3) is always much larger than all the rest terms.

How can the CSFE decay? This process is determined by two necessary conditions: by the availability of an interaction that does not conserve the electron system spin, and by a mechanism of energy dissipation making the relaxation process irreversible. Evidently, 
hyperfine coupling of electrons to GaAs nuclei is irrelevant here: this mechanism of spin non-conservation assumes that the irreversibility is realized in a purely electronic way - by exciton-exciton scattering processes reducing the total number of excitons. Due to energy conservation law and impossibility to compensate the energy of a decaying CSFE, the hyperfine scattering relaxation mechanism is forbidden. Just the same situation occurs if spin non-conservation is due to the spin-orbit coupling, provided that the irreversibility takes place due to an external smooth disordered potential or exciton-exciton Coulomb correlations [3]. The only CSFE relaxation channel is thus governed by the spin-orbit and electron-phonon couplings leading to the acoustic phonon emission. The relaxation probability is determined by the Fermi golden rule,

$$
w_{f i}=(2 \pi / \hbar)\left|\mathcal{M}_{f i}\right|^{2} \delta\left(E_{f}-E_{i}\right),
$$

where in the initial state $|i\rangle$ the number of SCFEs is by one larger than in the final $|f\rangle$, and contrariwise the number of phonons is lager by one in $|f\rangle$ than in $|i\rangle$, $\mathcal{M}_{f i}$ being the relevant matrix element.

Methodically it is useful to develop the approach as applied to the general case where $\nu=2 n+2(n=$ $0,1,2, \ldots)$. The spin-orbit coupling is described by a single electron term of the total Hamiltonian, namely:

$$
\begin{aligned}
\hat{H}_{\mathrm{so}} & =\alpha(\hat{\mathbf{q}} \times \hat{\boldsymbol{\sigma}})_{z}+\beta\left(\hat{q}_{y} \hat{\sigma}_{y}-\hat{q}_{x} \hat{\sigma}_{x}\right), \\
\hat{\mathbf{q}} & =-i \nabla+e \mathbf{A} / c \hbar .
\end{aligned}
$$

This operator, specified for the (001) GaAs plane, represents a combination of the Rashba term $(\sim \alpha)$ and the crystalline anisotropy term $(\sim \beta)[21,22]$ and does not violate translational symmetry. As usual [3, 7. 9. 17, 18] it is convenient to employ a bare singleelectron basis diagonalizing the single-electron Hamiltonian $\hat{\mathbf{q}}^{2} / 2 m_{e}^{*}+\hat{H}_{\mathrm{so}}$. To within the leading order in the $\hat{H}_{\text {so }}$ terms one obtains the basis states,

$$
\begin{aligned}
& \Psi_{n p}^{(a)}=\left(\begin{array}{c}
\psi_{n p} \\
v \sqrt{n+1} \psi_{n+1 p}+i u \sqrt{n} \psi_{n-1 p}
\end{array}\right) \text { and } \\
& \Psi_{n p}^{(b)}=\left(\begin{array}{c}
-v \sqrt{n} \psi_{n-1 p}+i u \sqrt{n+1} \psi_{n+1 p} \\
\psi_{n p}
\end{array}\right),
\end{aligned}
$$

where $u=\beta \sqrt{2} /{ }_{B} \hbar \omega_{c}$ and $v=\alpha \sqrt{2} /{ }_{B} \hbar \omega_{c}$ are small dimensionless parameters. Thus the single-electron states acquire a chirality $a$ or $b$ instead of spin-up and spin-down quantum numbers, and the spin flip actually corresponds to the $a \rightleftarrows b$ processes. The definition of the exciton creation operator formally remains the same [Eq. [1]], although the $a_{p}$ and $b_{p}$ operators describe annihilation in some of states (8) now. In particular, in the case of the CSFE $a_{p}$ corresponds to annihilation in the $\Psi_{n p}^{(b)}$ state and $b_{p}^{\dagger}$ to creation resulting in the $\Psi_{n+1 p}^{(a)}$ one. It is exactly this transition from $\Psi_{n p}^{(b)}$ to $\Psi_{n+1 p}^{(a)}$ that represents the $\bar{n} \rightarrow n+1$ promotion, and my task now is to calculate the relaxation of the $\mathcal{Q}_{\bar{n} n+1}^{\dagger} \mathbf{q}|0\rangle$ state.

The Hamiltonian of electron coupling to $3 \mathrm{D}$ acoustic phonons with momenta $\mathbf{k}=\left(\mathbf{q}, k_{z}\right)$ is written as

$$
\begin{aligned}
& \hat{H}_{\mathrm{e}-\mathrm{ph}}= \\
& \frac{\hbar^{1 / 2}}{L L_{z}^{1 / 2}} \sum_{\mathbf{q}, k_{z}, s} U^{\prime}{ }_{s}(\mathbf{k}) \hat{P}_{\mathbf{k}, s} \mathcal{H}_{\mathrm{e}-\mathrm{ph}}(\mathbf{q})+\text { H. c. }
\end{aligned}
$$

(see Refs. [7] and [17, 18] and references therein), where $L^{2}=2 \pi \mathcal{N}_{\phi} l_{B}^{2}$ is the $2 \mathrm{D}$ area, and $L_{z}$ is thickness of the slab along $\hat{z}$,

$$
\mathcal{H}_{\mathrm{e}-\mathrm{ph}}(\mathbf{q})=\int e^{i \mathbf{q r}} \hat{\Psi}^{\dagger}(\mathbf{r}) \hat{\Psi}(\mathbf{r}) d^{2} r+\text { H. c. } ;
$$

$\hat{P}_{\mathbf{k}, s}$ is the phonon annihilation operator (index $s$ denotes possible phonon polarizations),

$$
\hat{\Psi}(\mathbf{r})=\sum_{n p}\left[a_{n p} \Psi_{n p}^{(a)}+b_{n p} \Psi_{n p}^{(b)}\right],
$$

and $U^{\prime}{ }_{s}(\mathbf{k})$ is the renormalized vertex where integration with respect to $z$ has been already performed: $U^{\prime}{ }_{s}(\mathbf{k})=U_{s}(\mathbf{k}) \Phi\left(k_{z}\right)$. The form-factor is

$$
\Phi\left(k_{z}\right)=\int e^{i k_{z} z}|\chi(z)|^{2} d z
$$

Further manipulations are simplified in view of a basic feature of the studied relaxation process: only 'hard' phonons (with energy $\approx \hbar \omega_{c}$ ) are generated at the CSFE annihilation. Besides, the calculation shows that only phonons emitted almost parallel to the $\hat{z}$ direction are relevant, i. e.: $q \lesssim 1 \ll k_{z} \approx l_{B} \omega_{c} / s_{l}$. [As a result, the key point of the studied mechanism is an extreme smallness of the form-factor $\Phi\left(k_{z}\right)$ if calculated for wide quantum wells where in common units thickness is $d \simeq 20 \mathrm{~nm}$, and thus $k_{z} d \gg 1$. It is exactly this fact that determines the predicted very slow CSFE decay.] In this connection, there are also obvious simplifications for the vertex $U_{s}$, namely: (i) the polarization phonon field where the amplitude is inversely proportional to phonon wave vector $k$ may be ignored, therefore only contribution of the deformation phonon field where the amplitude is proportional to $\sqrt{k}$ has to be taken into account; (ii) only LA phonons $(s=l)$ 
give rise to the deformation potential in the GaAs lattice [23]. For the 3D vertex one needs only the expression for the square (c. f. Ref. [7]):

$$
\left|U_{l}\right|^{2}=\pi \varepsilon_{\mathrm{ph}}\left(k_{z}\right) / p_{0}^{3} \tau_{D},
$$

where the phonon energy is considered to be proportional to $k: \varepsilon_{\mathrm{ph}}(k)=\hbar s_{l} \sqrt{k_{z}^{2}+q^{2}} / l_{B}$ (I recall that $k_{z}$ and $\mathbf{q}$ are dimensionless), $p_{0}=2.52 \cdot 10^{6} \mathrm{~cm}^{-1}$ and $\tau_{D} \simeq 0.8 \mathrm{ps}$ are the material parameters of GaAs, (see Refs. [23] and [7]). The $\hat{z}$-direction corresponds to the direction $\Gamma \rightarrow \Delta \rightarrow X$ in the conjugate space, and therefore $s_{l} \approx 4.5 \cdot 10^{5} \mathrm{~cm} / \mathrm{s}$ [24].

So, the electron-phonon interaction model is completely determined by Eqs. (8)-(13). When substituting Eq. (11) into Eq. (10), I keep there only the terms governing the CSFE annihilation processes (i. e. the decay of the $\mathcal{Q}_{\bar{n} n+1}^{\dagger}|0\rangle$ state). As a result, the dimensionless operator $\mathcal{H}_{\mathrm{e}-\mathrm{ph}}$ in terms of excitonic representation relevant to the case takes the form:

$$
\mathcal{H}_{\mathrm{e}-\mathrm{ph}}(\mathbf{q})=\sqrt{\mathcal{N}_{\phi}} G_{n}(q) \mathcal{Q}_{\bar{n} n+1 \mathbf{q}}+\text { H. c. },
$$

where $G_{n}(q)=L_{n}^{1}\left(q^{2} / 2\right) e^{-q^{2} / 4}\left[v\left(q_{+}\right)^{2}+i u q^{2} / 2\right] /$ $(n+1)$ [here $q_{+}=-i\left(q_{x}+i q_{y}\right) / \sqrt{2}, L_{n}^{i}$ is the Laguerre Hamiltonian; in the derivation of Eq. (14) the equivalences $L_{n}^{2}(x)-L_{n-1}^{2}(x) \equiv L_{n}^{1}(x)$ and $L_{n}(x)-L_{n+1}(x) \equiv x L_{n}^{1}(x) /(n+1)$ have been used].

To calculate the transition matrix element $\mathcal{M}_{f i}$ in Eq. (6) one has to choose the initial and final states. These may correspond to a single exciton decay: $|i\rangle=\mathcal{Q}_{\bar{n} n+1 \mathbf{q}_{0}}^{\dagger}|0\rangle \rightarrow|f\rangle=\hat{P}_{\mathbf{k}, l}|0\rangle$, or to an exciton-exciton scattering process: $|i\rangle=$ $\mathcal{Q}_{\bar{n} n+1 \mathbf{q}_{1}}^{\dagger} \mathcal{Q}_{\bar{n} n+1 \mathbf{q}_{2}}^{\dagger}|0\rangle \rightarrow|f\rangle=\hat{P}_{\mathbf{k}, l} \mathcal{Q}_{\bar{n} n+1}^{\dagger} \mathbf{q}^{\prime}|0\rangle$. Respectively, the matrix element is equal to

$$
\begin{aligned}
& \mathcal{M}_{f i}^{(1)}\left(k_{z}, \mathbf{q}_{0}\right)=\frac{1}{l_{B}} \sqrt{\frac{\hbar}{2 \pi L_{z}}} \\
& \quad \times \sum_{\mathbf{q}} U_{l}\left(k_{z}\right) \Phi\left(k_{z}\right) G_{n}(q) M_{1}\left(\mathbf{q}, \mathbf{q}_{0}\right)
\end{aligned}
$$

or to

$$
\begin{aligned}
& \mathcal{M}_{f i}^{(2)}\left(k_{z}, \mathbf{q}^{\prime}, \mathbf{q}_{1}, \mathbf{q}_{2}\right)=\frac{1}{l_{B}} \sqrt{\frac{\hbar}{2 \pi L_{z}}} \\
& \quad \times \sum_{\mathbf{q}} U_{l}\left(k_{z}\right) \Phi\left(k_{z}\right) G_{n}(q) M_{2}\left(\mathbf{q}^{\prime}, \mathbf{q} ; \mathbf{q}_{1}, \mathbf{q}_{2}\right),
\end{aligned}
$$

where $M_{1,2}$ are expectations: $M_{1}=\left\langle 0\left|\mathcal{Q}_{\mathbf{q}} \mathcal{Q}_{\mathbf{q}_{0}}^{\dagger}\right| 0\right\rangle$ and $M_{2}=\left\langle 0\left|\mathcal{Q}_{\mathbf{q}^{\prime}} \mathcal{Q}_{\mathbf{q}} \mathcal{Q}_{\mathbf{q}_{1}}^{\dagger} \mathcal{Q}_{\mathbf{q}_{2}}^{\dagger}\right| 0\right\rangle$ (the subscripts are everywhere omitted, i.e. $\mathcal{Q}_{\mathbf{q}}$ actually stands for
$\left.\mathcal{Q}_{\bar{n} n+1 \mathbf{q}}\right)$. The expectations are calculated with the help of the commutation algebra 2 (see also Refs. [1. 3, 7,-9, 12-18]):

$$
\begin{aligned}
M_{1}=\delta_{\mathbf{q}, \mathbf{q}_{0}}, \quad M_{2}= & \delta_{\mathbf{q}^{\prime}, \mathbf{q}_{2}} \delta_{\mathbf{q}, \mathbf{q}_{1}}+\delta_{\mathbf{q}^{\prime}, \mathbf{q}_{1}} \delta_{\mathbf{q}, \mathbf{q}_{2}}- \\
& -\frac{2 \cos \phi}{\mathcal{N}_{\phi}} \delta_{\mathbf{q}_{1}+\mathbf{q}_{2}, \mathbf{q}^{\prime}+\mathbf{q}},
\end{aligned}
$$

where $\phi=\left(\mathbf{q}^{\prime} \times \mathbf{q}_{2}+\mathbf{q} \times \mathbf{q}_{1}\right)_{z} / 2$.

Equations (6)-(17) allow finding the decay probabilities $\sum_{f} w_{f i}$ of both states $\mathcal{Q}_{\mathbf{q}_{0}}^{\dagger}|0\rangle$ and $\mathcal{Q}_{\mathbf{q}_{1}}^{\dagger} \mathcal{Q}_{\mathbf{q}_{2}}^{\dagger}|0\rangle$. However, to calculate the CSFE relaxation rate one should know the distribution $N_{\mathbf{q}}$ of excitons over the q wave numbers. Although exciton operators (1) are non-bosonic, the excitons obey Bose statistics because their number in any state determined by a certain $\mathbf{q}$ may, in principle, be macroscopically large. At any moment the CSFE distribution is quasi-equilibrium, $N_{\mathbf{q}}=$ $1 /\left(e^{[E(1, \mathbf{q})-\mu] / T}-1\right)$, and characterized by chemical potential $\mu<E(1,0) \equiv \hbar \omega_{c}-\Delta E_{\mathrm{C}}-\epsilon_{\mathbf{Z}}$. (The thermodynamic equilibrium is certainly established much faster than the CSFE decay processes occur.) Initially the total number of excitons $\mathcal{N}_{\mathbf{x}}=\sum_{\mathbf{q}} N(\mathbf{q})$ is actually determined by a short external optical pulse. It may be rather large, though still appreciably smaller than $\mathcal{N}_{\phi}$. For a given number $\mathcal{N}_{\mathbf{x}}$ one can find $\mu$, and using Eq. (3) obtain

$$
N_{\mathbf{q}}=\frac{1-e^{-n_{\mathrm{x}} / M_{\mathrm{x}} T}}{e^{q^{2} / 2 M_{\mathrm{x}} T}-1+e^{-n_{\mathrm{x}} / M_{\mathrm{x}} T}},
$$

where $n_{\mathrm{x}}=\mathcal{N}_{\mathrm{x}} / \mathcal{N}_{\phi}$ is the CSFE concentration.

Now, with the help of Eq. (6) and Eqs. (13)-(18) one can calculate the relaxation rate, $R=\sum_{f, i} w_{f i}$. In the case of single-exciton relaxation it yields

$$
\begin{aligned}
& R_{1}= \\
& \frac{2 \pi}{\hbar} \sum_{k_{z}, \mathbf{q}_{0}}\left|\mathcal{M}_{f i}^{(1)}\left(\mathbf{q}_{0}\right)\right|^{2} \delta\left(\hbar \omega_{c}-\hbar s_{l} \sqrt{k_{z}^{2}+q_{0}^{2}} / l_{B}\right) \approx \\
& \frac{\left|U_{l}\left(\omega_{c} / s_{l}\right) \Phi\left(\omega_{c} / s_{l}\right)\right|^{2}}{\pi \hbar l_{B}^{2} s_{l}} \sum_{\mathbf{q}_{0}} N_{\mathbf{q}_{0}}\left|G_{n}\left(q_{0}\right)\right|^{2}
\end{aligned}
$$

As a result, the equation governing temporal dependence of the CSFE concentration, $-d n_{\mathrm{x}}(t) / d t=$ 
$=R_{1} / N_{\phi}$, after performing summation $\sum_{\mathbf{q}} \ldots=N_{\phi}$

$\times \int \ldots q d q$ takes the following form:

$$
-\frac{d\left(n_{\mathrm{x}} / M_{\mathrm{x}} T\right)}{d t}=\frac{1}{\tau_{1}} \sum_{m=1}^{\infty} m^{-3}\left(1-e^{-n_{\mathrm{x}} / M_{\mathrm{x}} T}\right)^{m}
$$

where

$$
\frac{1}{\tau_{1}}=\frac{2 \omega_{c}\left|M_{\mathrm{x}} T \Phi\left(\omega_{c} / s_{l}\right)\right|^{2}\left(u^{2}+v^{2}\right)}{p_{0}^{3} l_{B}^{2} s_{l} \tau_{D}} .
$$

The inverse time $1 / \tau_{1}$ vanishes if $T \rightarrow 0$. This means, in particular, that the relaxation at $n_{\mathrm{x}}>M_{\mathrm{x}} T$ is determined by the exciton-exciton scattering, that is by the rate

$$
\begin{aligned}
R_{2}= & \frac{\pi}{\hbar} \sum_{k_{z}, \mathbf{q}^{\prime}, \mathbf{q}_{1}, \mathbf{q}_{2}}\left|\mathcal{M}_{f i}^{(2)}\left(k_{z}, \mathbf{q}^{\prime}, \mathbf{q}_{1}, \mathbf{q}_{2}\right)\right|^{2} \\
& \times \delta\left(\hbar \omega_{c}-\hbar s_{l} \sqrt{k_{z}^{2}+q^{\prime 2}} / l_{B}\right) \\
\approx & \frac{2\left|U_{l}\left(\omega_{c} / s_{l}\right) \Phi\left(\omega_{c} / s_{l}\right)\right|^{2}}{\pi \hbar l_{B}^{2} s_{l} \mathcal{N}_{\phi}^{2}} \\
& \times \sum_{\mathbf{q}_{1}, \mathbf{q}_{2}, \mathbf{q}^{\prime}} N_{\mathbf{q}_{1}} N_{\mathbf{q}_{2}}\left|G_{n}\left(\left|\mathbf{q}_{1}+\mathbf{q}_{2}-\mathbf{q}^{\prime}\right|\right)\right|^{2} .
\end{aligned}
$$

Here the relevant values of $q_{1}$ and $q_{2}$ are taken into account to be of the order of $\sqrt{M_{\mathrm{x}} T} \ll 1$. Therefore the dominant contribution to the rate is provided by the $\sim$ $1 / \mathcal{N}_{\phi}$ term of the second expectation $(17)$, where also $\phi \approx 0$ has to be set. Besides, the $\sum_{\mathbf{q}_{1}, \mathbf{q}_{2}, \mathbf{q}^{\prime}} \ldots$ summation in Eq. 22 is reduced to $\sum_{\mathbf{q}_{1}, \mathbf{q}_{2}} N_{\mathbf{q}_{1}} N_{\mathbf{q}_{2}} \sum_{\mathbf{q}^{\prime}}\left|G_{n}\left(q^{\prime}\right)\right|^{2}=$ $2 N_{\phi} N_{\mathrm{x}}^{2}\left(u^{2}+v^{2}\right)$. Finally, the equation $-d n_{\mathrm{x}}(t) / d t=$ $R_{2} / N_{\phi}$ takes form $-d n_{\mathrm{x}} / d t=n_{\mathrm{x}}^{2} / \tau_{2}$ with characteristic inverse relaxation time

$$
\frac{1}{\tau_{2}}=\frac{4 \omega_{c}\left|\Phi\left(\omega_{c} / s_{l}\right)\right|^{2}\left(u^{2}+v^{2}\right)}{p_{0}^{3} l_{B}^{2} s_{l} \tau_{D}},
$$

The SCFE density thus decays non-exponentially, obeying the equation $n_{\mathrm{x}}(t)=n_{\mathrm{x}}(0) /\left(1+n_{\mathrm{x}}(0) t / \tau_{2}\right)$. The relaxation time $\tau_{2}$ is shorter than $\tau_{1}$, differing by a small factor $\left(M_{\mathrm{x}} T\right)^{2} / 2 \sim 10^{-3}-10^{-4}$. This feature is a consequence of the phase volume enhancement in the case of the exciton-exciton scattering. Yet note that due to the non-exponentiality of the relaxation, the real value which should be compared with experimental results is not $\tau_{2}$ but $\tau_{2}^{\prime} \sim \tau_{2} / n_{\mathrm{x}}(0)$. If the CSFE is excited optically, relevant magnitudes of the initial density are $n_{\mathrm{x}}(0) \sim 0.01$.

I focus on numerical estimates of $\tau_{2}$. When so doing, one faces the basic difficulty related to uncertainty in the $\Phi\left(\omega_{c} / s_{l}\right)$ value. This one strongly depends on the poorly observable function $\chi(z)$. However, it is clear that for a wide quantum well with effective thickness $d \simeq 20 \mathrm{~nm}$ and for the magnetic field, e. g. equal to $5 \mathrm{~T}$, a considerable incommensurability of $d$ and $\omega_{c} / s_{l}=k_{z} \simeq 3 / \mathrm{nm}$ takes place. This fact extremely reduces the Fourier component of $|\chi(z)|^{2}$ [Eq. [12)]. When estimating $\chi(z)$ by means of three models: (i) of Fang and Howard; (ii) of Takada and Uemura (see Ref. [25] and references therein); (iii) of the simple model where quantum-well walls are considered to be infinitely high, one finds that $|\Phi(k)|^{2} \approx \mathcal{C} /(k d)^{6}$ where $\mathcal{C} \approx 4.7 \cdot 10^{4}, 6.5 \cdot 10^{3}$, and $3.1 \cdot 10^{3}$ respectively. $(d / 2$ in all three models is set equal to the average penetration length of the charge into the semiconductor.) Substitution of this estimate into Eq. (23) and assumption that $u^{2}+v^{2}=10^{-3} / B$ ( $B$ is in Teslas) [7. -9] yields for the quantum well with $d=20 \mathrm{~nm}$ : $\tau_{2} \approx 10^{-2} B^{5} / \mathcal{C}$, and hence $\tau_{2}^{\prime} \sim B^{5} / \mathcal{C}$. At $B=5 \mathrm{~T}$ even for the 'fastest' Fang-Howard model describing the $\chi(z)$ wave-function, one gets estimates $\sim 7 \mathrm{~ms}$ and $\sim 0.7 \mathrm{~s}$ for the $\tau_{2}$ and $\tau_{2}^{\prime}$ times respectively.

I concern also the relaxation scenarios of other excitations in this system with energies close to the same energy $\hbar \omega_{c}$. First, there is a spinless magnetoplasma mode where electron is promoted from the zero Landau level to the first one without any spin change [it is described excitonically as $\left.2^{-1 / 2}\left(\mathcal{Q}_{\overline{01}}^{\dagger}+\mathcal{Q}_{01}^{\dagger}\right)|0\rangle\right]$. Estimates reveal that this exciton should have a considerable radiative relaxation channel through emission of a photon with energy $\hbar \omega_{c}$. For example, at $B=5 \mathrm{~T}$ the magnetoplasma mode decays within $\sim 10-100 \mu \mathrm{s}$. Second, there are two other components of the CSFE triplet. These are higher by $\epsilon_{Z}$ and $2 \epsilon_{Z}$ than the studied lowest energy component of the CSFE, and have spin numbers $S_{z}=0$ and -1 respectively, see Eq. (3). [Excitonically, these are $2^{-1 / 2}\left(\mathcal{Q}_{\overline{01}}^{\dagger}-\mathcal{Q}_{01}^{\dagger}\right)|0\rangle$ and $\mathcal{Q}_{0 \overline{1}}^{\dagger}|0\rangle$ excitons]. Both modes obviously relax in a cascade manner by two stages. The initial stage is fast and governed by the exciton-exciton scattering, leading to conversion of one of the excitons into the lowest CSFE component. Calculation of this scattering rate should account for several possible relaxation mechanisms (including emitting of a phonon with energy $\sim \epsilon_{\mathrm{Z}}$ ) similar to those considered for spin-waves in a quantum Hall ferromagnet [7]. The dominant process depends on specific parameters $B$ and $T$; however, in any case the relevant relaxation time turns out to be shorter than $1 \mu$ s, i. e. much smaller than $\tau_{2}$. Thus, due to fast processes the $S_{z}=0$ and $S_{z}=-1$ modes convert into the 
$S_{z}=1$ metastable mode. The latter for its part relaxes slowly in the above studied way.

To sum up, I have studied relaxation of the lowestenergy excitation in the unpolarized even-integer quantum Hall system. This excitation, representing a cyclotron spin-flip mode, should actually be metastable because even the fastest relaxation mechanism results in the characteristic life time of the order of $1 \mathrm{~s}$. The estimate should be valid for wide quantum wells (where thickness is $>20 \mathrm{~ns}$ ) and for magnetic fields higher than $5 \mathrm{~T}$. In fact, the studied relaxation is simultaneously the energy and spin relaxation process and, if compared to an extremely long spin relaxation in single-electron quantum dot [10], turns out even longer than the latter. Meanwhile note that the filling factor deviation from the number 2 (or $4,6, \ldots$ ) should substantially accelerate the CSFE relaxation, because appearance of effective holes in the zero Landau level (if $\nu<2$ ), or electrons in the first one (if $\nu>2$ ) will increase the phase volume of the relaxation processes.

The author acknowledges support of the Russian Fund of Basic Research.

\section{References}

[1] S. Dickmann and I.V. Kukushkin, Phys. Rev. B 71, 241310(R) (2005), http://dx.doi.org/10.1103/PhysRevB.71.241310

[2] L.V. Kulik, I.V. Kukushkin, S. Dickmann, V.E. Kirpichev, A.B. Van'kov, A.L. Parakhonsky, J.H. Smet, K. von Klitzing, and W. Wegscheider, Phys. Rev. B 72, 073304 (2005), http://dx.doi.org/10.1103/PhysRevB.72.073304

[3] S. Dickmann and S.L. Artyukhin, JETP Lett. 89, 133 (2009), http://dx.doi.org/10.1134/S0021364009030084

[4] D. Fukuoka, K. Oto, K. Muro, Y. Hirayama, and N. Kumada, Phys. Rev. Lett. 105, 126802 (2010), http://dx.doi.org/10.1103/PhysRevLett.105.126802

[5] D. Fukuoka, T. Yamazaki, N. Tanaka, K. Oto, K. Muro, Y. Hirayama, N. Kumada, and H. Yamaguchi, Phys. Rev. B 78, 041304(R) (2008), http://dx.doi.org/10.1103/PhysRevB.78.041304

[6] Yu.A. Nefyodov, A.A. Fortunatov, A.V. Shchepetilnikov, and I.V. Kukushkin, JETP Lett. 91, 357 (2010), http://dx.doi.org/10.1134/S0021364010070076
[7] S. Dickmann and T. Ziman, Phys. Rev. B 85, 045318 (2012), http://dx.doi.org/10.1103/PhysRevB.85.045318

[8] S. Dickmann, Phys. Rev. Lett. 93, 206804 (2004), http://dx.doi.org/10.1103/PhysRevLett.93.206804

[9] S. Dickmann, JETP Letters 93, 86 (2011), http://dx.doi.org/10.1134/S0021364011020068

[10] S. Amasha, K. MacLean, I.P. Radu, D.M. Zumbühl, M.A. Kastner, M.P. Hanson, and A.C. Gossard, Phys. Rev. Lett. 100, 046803 (2008), http://dx.doi.org/10.1103/PhysRevLett.100.046803

[11] C. Kallin and B.I. Halperin, Phys. Rev. B 30, 5655 (1984), http://dx.doi.org/10.1103/PhysRevB.30.5655

[12] S. Dickmann and V.M. Zhilin, Phys. Rev. B 78, 115302 (2008), http://dx.doi.org/10.1103/PhysRevB.78.115302

[13] L.V. Kulik, S. Dickmann, I.K. Drozdov, I.S. Zhuravlev, V.E. Kirpichev, I.V. Kukushkin, S. Schmult, and W. Dietsche, Phys. Rev. B 79, 121310(R) (2009), http://dx.doi.org/10.1103/PhysRevB.79.121310

[14] S. Dickmann and Y. Levinson, Phys. Rev. B. 60, 77607763 (1999), http://dx.doi.org/10.1103/PhysRevB.60.7760

[15] S. Dickmann and Y. Levinson, Physica E 5, 153-156 (2000), http://dx.doi.org/10.1016/S1386-9477(99)00255-6

[16] S. Dickmann, Phys. Rev. B 61, 5461-5473 (2000), http://dx.doi.org/10.1103/PhysRevB.61.5461

[17] S.M. Dikman and S.V. Iordanskii, JETP Lett. 63, 50 (1996), http://dx.doi.org/10.1134/1.566962

[18] S. Dickmann and S.V. Iordanskii, JETP 83, 128 (1996)

[19] A.B. Dzyubenko and Yu.E. Lozovik, Sov. Phys. Solid State 25, 874 (1983)

[20] A.B. Dzyubenko and Yu.E. Lozovik, Sov. Phys. Solid State 26, 938 (1984)

[21] Yu.A. Bychkov and E.I. Rashba, JETP Lett. 39, 78 (1984)

[22] M.I. D’yakonov and V.Yu. Kachorovskii, Sov. Phys. Semicond. 20, 110 (1986)

[23] V.F. Gantmakher, and Y.B. Levinson, Carrier Scattering in Metals and Semiconductors (North-Holland, Amsterdam, 1987)

[24] D. Strauch and B. Dorner, J. Phys. Condens. Matter 2, 1457 (1990), http://dx.doi.org/10.1088/0953-8984/2/6/006

[25] T. Ando, A.B. Fowler, and F. Stern, Rev. Mod. Phys. 54, 437 (1982), http://dx.doi.org/10.1103/RevModPhys.54.437 